\title{
Genomic evidence of a SARS-CoV-2 reinfection case with E484K spike mutation in Brazil
}

Carolina Kymie Vasques Nonaka ${ }^{1,2}$, Marília Miranda Franco ${ }^{3}$, Tiago Gräf ${ }^{3}$, Ana Verena Almeida Mendes ${ }^{4}$, Renato Santana de Aguiar ${ }^{5}$, Marta Giovanetti ${ }^{6,78^{*}} \&$ Bruno Solano de Freitas Souza $^{1-3^{*}}$

${ }^{1}$ Center for Biotechnology and Cell Therapy, São Rafael Hospital, Salvador, Brazil; ${ }^{2}$ D'Or Institute for Research and Education (IDOR), Rio de Janeiro, Brazil; ${ }^{3}$ Gonçalo Moniz Institute, FIOCRUZ, Salvador, Brazil; ${ }^{4}$ Department of Infectology, São Rafael Hospital, Salvador, Brazil; ${ }^{5}$ Department of Genetics, Ecology and Evolution, Institute of Biological Sciences, Universidade Federal de Minas Gerais, Belo Horizonte, Brazil; ${ }^{6}$ Laboratório de Flavivírus, Instituto Oswaldo Cruz, Fundação Oswaldo Cruz, Rio de Janeiro, Brazil; ${ }^{7}$ Laboratório de Genética Celular e Molecular, Instituto de Ciências Biológicas, Universidade Federal de Minas Gerais, Belo Horizonte, Minas Gerais, Brazil; ${ }^{8}$ Unit of Medical Statistics and Molecular Epidemiology, University Campus Bio-Medico of Rome, Rome, Italy.

*Correspondence:

Dr. Bruno S. F. Souza, MD, PhD, bruno.solano@fiocruz.br; Dr. Marta Giovanetti, PhD, giovanetti.marta@gmail.com

Keywords: SARS-CoV-2; reinfection; Brazil; E484K

\begin{abstract}
To date, uncertainty remains about how long the protective immune responses against SARS$\mathrm{CoV}-2$ persists and the first reports of suspected reinfection began to be described in recovered patients months after the first episode. ${ }^{1}$ Viral evolution may favor reinfections, and the recently described spike mutations, particularly in the receptor binding domain (RBD) in SARS-CoV2 lineages circulating in the UK, South Africa, and most recently in Brazil, have raised concern on their potential impact in infectivity and immune escape. ${ }^{2,3,4} \mathrm{We}$ report the first case of reinfection from genetically distinct SARS-CoV-2 lineage presenting the E484K spike mutation in Brazil, a variant associated with escape from neutralizing antibodies.
\end{abstract}




\section{Text}

A 45-year-old female healthcare executive, resident in Salvador, Bahia state, Northeast Brazil, with no comorbidities, presenting symptoms of viral infection on two occasions (May 26, 2020 and October 26, 2020). In the first episode, the patient presented diarrhea, myalgia, asthenia and odynophagia for approximately 7 days. She used $40 \mathrm{mg}$ prednisone for 5 days and returned to activities 21 days later without sequelae or complaints. In the second episode, symptomatically more severe, the patient presented headache, malaise, diarrhea, cough and sore throat that evolved to myalgia and respiratory distress, ageusia, muscle fatigue, insomnia, mild dyspnea on exertion and shortness of breath. In both occasions, results of RT-PCR tests targeting 3 genes (N, E and RdR) were positive for SARS-CoV-2 in nasopharyngeal samples (Figure 1A). Cycle threshold values (Cts) of N, E and RdRp targets were 25, 26, and 27 in the first episode and 21, 12 and 17 in the second episode. Four weeks after testing positive by RTPCR in the second episode, an IgG test was performed and showed a positive result (index value: 2.15 on $11 / 23 / 2020)$.

Sequencing was conducted on the two nasopharyngeal swabs by PGM Ion Torrent (Life Technologies, USA) according to the manufacturer's instructions. Sequence alignment and phylogenetic analysis were performed as previously described. ${ }^{3}$ Sequencing statistics showed 1.405.009 mapped reads for sample A and 2.570.182 reads for sample B, resulting in a sequencing mean depth $>1,000 \mathrm{X}$ for both samples and a coverage $>99 \%$. Using the pangolin COVID-19 lineage Assigner (https://pangolin.cog-uk.io/), sample A was identified as B.1.1.33 lineage and sample B as B.1.1.248, a lineage derived from B.1.1.28, recently identified in Brazil. ${ }^{4}$ Phylogenetic analysis, of the two newly whole genome sequences compared with contemporaneous sequences from Brazil (supplementary table 1), clearly demonstrated that the two COVID-19 episodes, separated by a 147-day interval, were indeed caused by different SARS-CoV-2 lineages, confirming reinfection (Figure 1B).

Moreover, we identified several mutations distinguishing the two genomes (Figure 1C), three of them in the SARS-CoV-2 spike glycoprotein. In the first infection, the retrieved genome presented the S:G1219C, while in the second infection, S:E484K and S:V1176F were observed. In both samples, we found ORF1ab:P4715L, S:D614G, N:R203K, and N:G204R mutations.

Mutation analysis demonstrated, for the first time, a reinfection case with a viral variant harboring the mutation $\mathrm{E} 484 \mathrm{~K}$, located in a key residue of the receptor binding domain, that 
seems to modestly enhance binding between the Spike protein and the host receptor hACE2. The E484K mutation is part of a group of variants identified in South Africa that have been associated with increased infectivity and was also observed as occurring in the recently described B.1.1.248 lineage in Brazil., ${ }^{3,4}$ This lineage was initially described in samples from Rio de Janeiro, but was already detected in UK, USA, Portugal and Australia. Here we show that B.1.1.248 may be widely spread among Brazilian states as well. The RBD is also a major target of neutralizing antibodies elicited during the primary exposure to SARS-CoV-2 and the E484K mutation has been predicted to affect antibody neutralization. ${ }^{5}$ The finding of the $\mathrm{E} 484 \mathrm{~K}$, in an episode of SARS-CoV-2 reinfection might have major implications for public health policies, surveillance and immunization strategies.

\section{Declaration of interest and funding source}

We declare no competing interests. This work was supported by grants provided by INOVA Fiocruz, Serrapilheira Institute and IDOR. 


\section{References}

1. Tillett RL, Sevinsky JR, Hartle PD, et al. Genomic evidence for reinfection with SARS-CoV2: a case study. Lancet Infect Dis 2020; 21: 52-58.

2. Rambaut A, Loman N, Pybus $\mathrm{O}$, et al. Preliminary genomic characterisation of an emergent SARS-CoV-2 lineage in the UK defined by a novel set of spike mutations. https://virological.org/t/preliminary-genomic-characterisation-of-an-emergent-sars-cov-2lineage-in-the-uk-defined-by-a-novel-set-of-spike-mutations/563. (accessed Dec 21, 2020).

3. Tegally H, Wilkinson E, Giovanetti M, et al. Emergence and rapid spread of a new severe acute respiratory syndrome-related coronavirus 2 (SARS-CoV-2) lineage with multiple spike mutations in South Africa. medRxiv 2020.12.21.20248640 (Preprint); doi: https://doi.org/10.1101/2020.12.21.20248640.

4. Voloch CM, Silva F R da, de Almeida LGP, et al. Genomic characterization of a novel SARSCoV-2 lineage from Rio de Janeiro, Brazil. medRxiv 2020.12.23.20248598 (Preprint). doi: https://doi.org/10.1101/2020.12.23.20248598

5. Thomson EC, Rosen LE, Shepherd JG, et al. The circulating SARS-CoV-2 spike variant N439K maintains fitness while evading antibody-mediatedimmunity.bioRxiv 2020:2020.2011.2004.355842 (Preprint); doi: https://doi.org/10.1101/2020.11.04.355842 . 


\section{Figure legend}

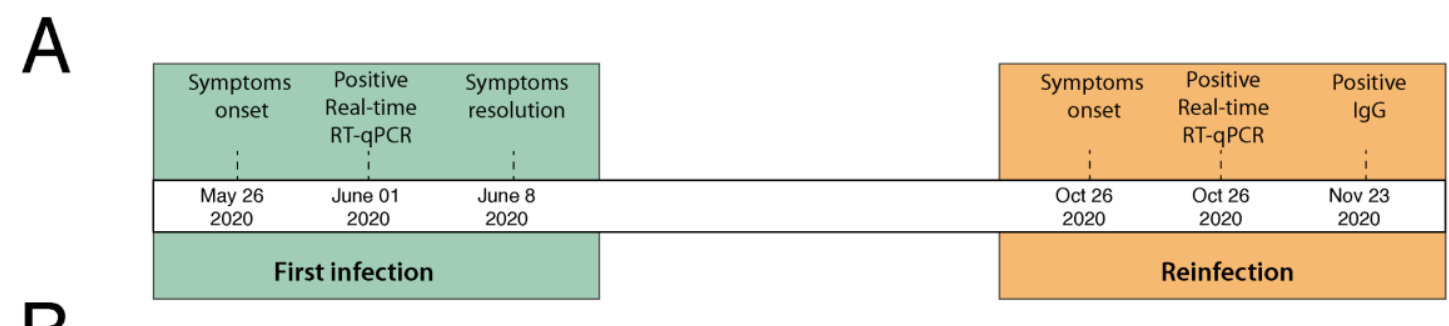

B

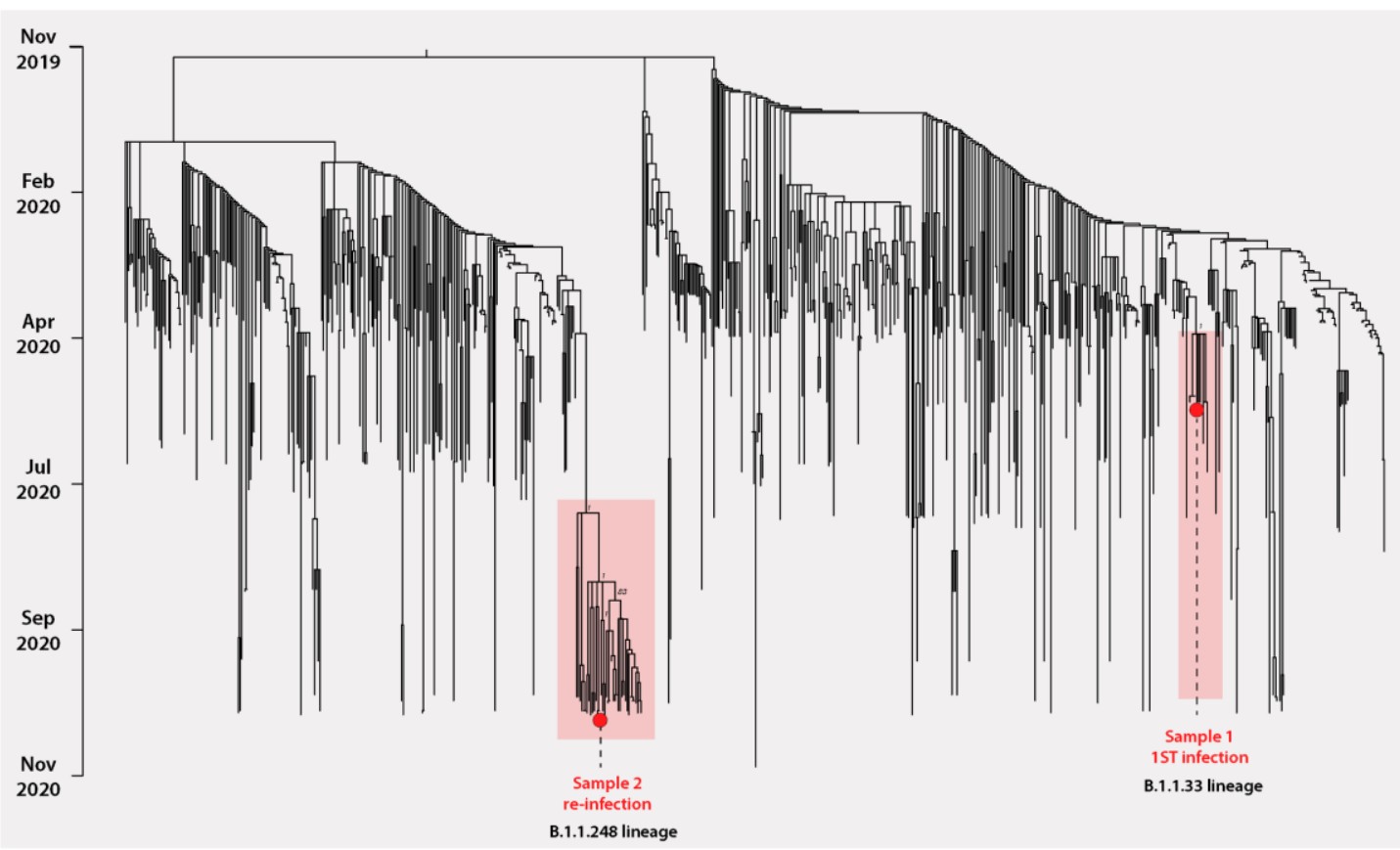

C

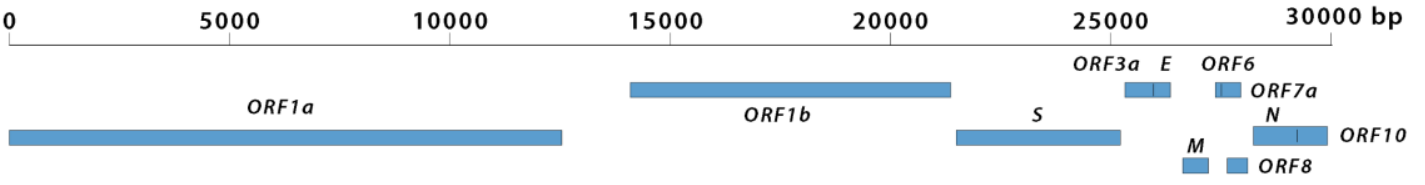

\begin{tabular}{ccc}
\multicolumn{3}{c}{ Mutational pattern sample 1 } \\
\hline Gene & Nucleotide & Amino acid \\
\hline ORF1ab & C4575T & T1437/ \\
& C14408T & P4715L \\
Spike & A23403G & D614G \\
& G25217T & G1219C \\
ORF6 & T27299C & I33T \\
& G28881A e & R203K \\
N & G28882A & G204R \\
& G28883C & I292T \\
\hline
\end{tabular}

\begin{tabular}{ccc}
\multicolumn{3}{c}{ Mutational pattern sample 2} \\
\hline Gene & Nucleotide & Amino acid \\
\hline ORF1ab & T10667G & L3468V \\
& C12053T & \\
& C14408T & P4715L \\
Spike & G23012A & E484K \\
& A23403G & D614G \\
& G25088T & V1176F \\
N & G28628T & A119S \\
& G28881A e & \\
& G28882A & R203K \\
& G28883C & G204R \\
& C28892T & P207S \\
& G28975T & M234I \\
\hline
\end{tabular}

Figure 1. Molecular characterization of a COVID-19 reinfection case in Salvador, Bahia state, Northeast Brazil. A) Timeline of symptom onset, molecular and serological diagnosis. B) Time-scaled ML tree including the newly SARS-CoV-2 genomes recovered from a 45-yearold female resident in Salvador, Bahia state, Northeast Brazil, plus contemporaneous sequences from Brazil (supplementary table 1). New genomes are highlighted with red circles. Support 
for branching structure is shown by bootstrap values at nodes. C) Mutational pattern of the two isolates obtained from the same patient with a 147-day interval. Mutations distinguishing the two infections are highlighted.

\section{Acknowledgments}

Authors thank Vagner Fonseca for the bioinformatics assessment. 